\section{A Suggested Scheme for the Investigation of} Marine Bacteria.

By

H. S. Holden, M.Sc., F.L.S.

Bacteriologist (Temporary), R.N. Hospital, Plymouth.

With one Figure in the Text.

WhILST employed at the Royal Naval Hospital, Plymouth, I have been kindly granted facilities for the investigation of marine bacteria by Dr. E. J. Allen, Director of the Marine Biological Laboratories, and although the pressure of other duties has made progress extremely slow the experience obtained with different media will perhaps be of use to future workers. After a number of trials the three routine media found to be most satisfactory were :-

1. Blood glucose agar, a slight modification of an opaque medium devised by Dr. Warren Crowe whilst investigating the epidemic of Cerebro-spinal Fever at the Devonport Military Hospital.

2. Trypsin Agar.

3. Trypsin Broth.

Since the trypsin broth enters into the composition of both 1 and 2 its preparation will be described first. The fat is removed from a fresh bullock's heart and the lean muscle is passed through a mincing machine. The minced muscle is placed in a saucepan with four litres of water and the whole rendered faintly alkaline to litmus with $40 \% \mathrm{NaOH}$. It is then slowly heated to $70^{\circ}-80^{\circ} \mathrm{C}$. and allowed to cool to $45^{\circ} \mathrm{C}$. Forty cubic centimetres of Liquor Trypsin Co. (Allen and Hanbury) are added to the warm broth, which is placed, after thorough mixing, in the incubator at $37^{\circ} \mathrm{C}$. for $2 \frac{1}{2}-3$ hours to trypsinise. After removal from the incubator the broth, if not already so, is rendered faintly acid to litmus with acetic acid. It is then raised to the boiling point, filtered through sterile muslin into a large vessel and rendered slightly alkaline to litmus. The filtrate is returned to a clean saucepan, and, after the addition of 10 grammes of $\mathrm{NaCl}$ and 0.5 gramme of $\mathrm{CaCl}_{2}$, again raised to the boiling point.
It is finally filtered into sterile flasks, autoclaved on three successive days, and stored in the dark.

Trypsin Agar is a 2\% Agar made up with trypsin broth, and clarified and filtered in the usual way.

The Blood Glucose Agar consists of defibrinated bullock's blood* to which trypsin agar and glucose have been added. The blood is collected

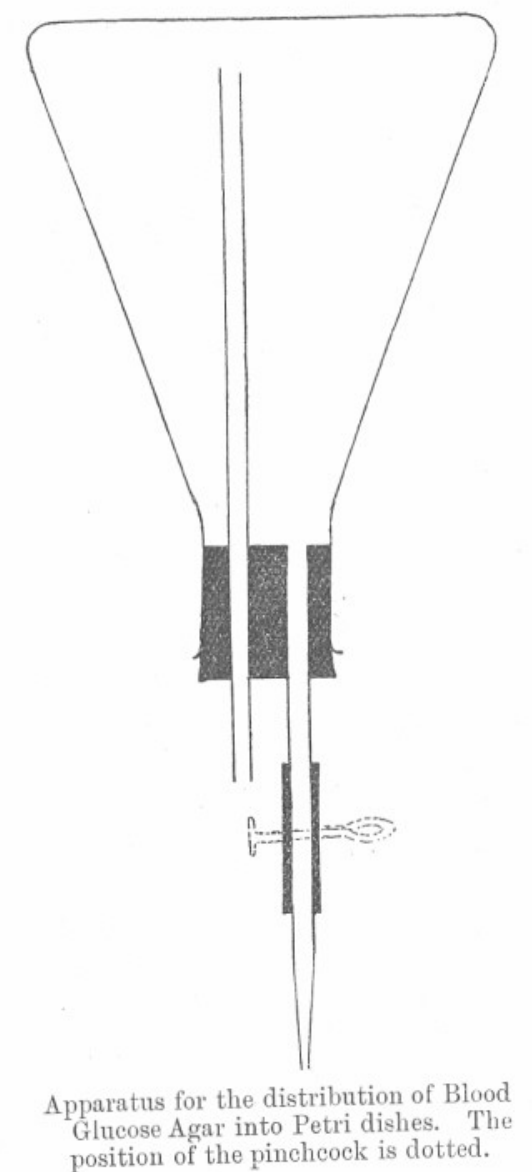

by an assistant from the abbatoir in a large enamel pail which, just prior to is defibrinated to the collection, is thor minutes continuous whisking being with a large wire whisk, about the major portion of the clot. Whilst this prammes of glucose and 25 cubic part of the preparation is under watecentimetres of distilled water are placed in a two-litre flask and autoclaved to dissolve the glucose. At the sab cen 
of trypsin agar and the necessary delivery tubes, stopper, and pinch cock (vide diagram), the latter previously put together and wrapped in a cloth, are also autoclaved. On reaching the laboratory a litre of the defibrinated blood is filtered through sterile gauze into the flask containing the glucose solution, which is then warmed in a bowl of water to $56^{\circ} \mathrm{C}$. being shaken gently at intervals. The agar at the same time is cooled to $56^{\circ} \mathrm{C}$. and then added to the blood and glucose and mixed thoroughly. The sterilised two-way stopper with the tubes and pinch cock as indicated in the diagram is then fitted and the flask promptly inverted and supported in a cloth-covered iron ring on a retort stand. The medium is distributed fairly deeply into sterile petri-dishes each with a tightly fitting filter paper in the lid. It is sterilised at $70^{\circ} \mathrm{C}$. for 3-4 hours on the first day and on the second day heated slowly (four hours) to $80^{\circ} \mathrm{C}$. The plates are then inverted and stored in the dark. The two most important points are thorough defibrination and thorough mixing of the blood with the agar, failure in the second respect entailing the formation of more or less extensive serum blisters. A good sample should have the appearance of a high-grade milk chocolate and have a slightly soft rubbery touch. If too hard it is unsuitable. This medium is admittedly slightly tedious to make up but its value is undoubted Working with translucent media such as agar and gelatin one is faced by a bewildering number of approximately white colonies and the surface and margin characters are the only macroscopic means of readily differentiating them. With the opaque blood medium, on the other hand, emphasis is given to slight differences both in colour and transparency, with the further advantage that many organisms react with the haemoglobin and acquire a characteristic coloration varying from shell pink to brown.

The routine I have adopted is to inoculate a number of plates with $0.05 \mathrm{cc}$. of sea-water taken, with a capillary pipette, from just below the surface of a selected tank and distributed over the surface with a small glass 'spreader.' The plates are incubated at $20^{\circ} \mathrm{C}$. for $3-5$ days and colonies which are common to a number are sub-cultured on to two agar slopes and into trypsin broth, a record being made of the size, configuration, margin, consistency and colour of the primary growth.

From the broth culture the following observations are made after 48 hours' incubation :-

1. A loopful is mounted as a hanging drop to determine form and motility.

2. A loopful is placed in the centre of a clean cover slip, held for a moment in the fumes of $1 \%$ osmic acid and then inverted on a clean slide. Measurements are carried out on this material and variations in length, etc. noted.
The culture is then left undisturbed to study the nature of the continued growth, e.g. the development or not of uniform turbidity, sedimentation, the formation of flocculi, development or not of a surface scum.

If the organism is motile a film is made from a 24-hour culture on gar. The method which has given the best results is that of Stephens.* agar. The meth of water in a watch glass and from this a very small loopful is transferred to a minute drop of water on an absolutely clean grease-free slide, mixed and spread at once. If this operation has been properly done the film should dry once. If this operation has been property dollowing :immediately. It is then mordanted for 1-2 minutes in the fol

$$
\begin{aligned}
& 2 \% \text { Aqueous Solution of Osmic Acid } 1 \text { part. } \\
& 20 \% \quad \text { ", Tannin } 3-4 \text { parts. }
\end{aligned}
$$

after which it is washed thoroughly in running water and drained well.

few drops of silver nitrate solution (silver nitrate crystals, 1 gramme

A few dre film for a few seconds and the water 100 cc.) excess shaken off. A drop of reducing solution (2\% aqueous solution of gallic acid, 1 part; ammonia fort., 1 part) is placed on the centre of the film which rapidly blackens. As soon as blackening is complete the film which rapidy solution for about rinse in tap water, pour on a few drops of the silver solution for

30 seconds, rinse again in tap water, blot and dry over a flame.

From the agar slopes the following sub-cultures are recommended as a routine :-

1. Gelatin stab. Note the nature of the growth and of liquefaction if present.

2. Potato.

3. Carbohydrate media. These consist of peptone water with the addition of the appropriate carbokydrate and an indicator. addition of the appropriate carber to collect some of the gas A Durham's tube is usually inserted to collect some of the formula :-
given off. Besson† gives the following general form

$\begin{array}{ll}\text { Peptone } & 1-2 \text { grammes. } \\ \text { Water } & 100 \mathrm{cc} . \\ \text { Test substance } & 1 \text { gramme. } \\ \text { Litmus } & \text { Quant. suff. }\end{array}$

Many carbohydrates are at present either unobtainable or cost proitive sums, but glucose, lactose, maltose, saccharose and mannite Lately I have used acid fuchsin $0.5 \%$ solution andicator and find it distinctly preferable to litmus as it gives very * Besson, A., Practical Bacteriology, Microbiology and Serum Therapy, trans. H. J. Hutchens, pp. 153-154, pub. Longmans and Co.

$\dagger$ Loc. cit., pp. 34-35. 
sharp readings. The peptone water and added carbohydrate are rendered slightly alkaline with normal $\mathrm{NaOH}$ and $1 \%$ of the indicator added. Hot titration with $\mathrm{N} / 10 \mathrm{HCl}$ is employed, the acid being added cautiously until a faint pink colour appears. The medium is colourless when cold, and becomes bright red after inoculation with an acid producing organism.

4. Gran's medium or Drew's modification of this to study nitrate reduction if present.*

Weak dilutions of emulsified growth in filtered, sterilised sea-water should also be plated out on agar and gelatin plates and the character of the discrete colonies noted.

(A film should be made and stained by Gram's method.)

I should finally suggest a working knowledge of the following organisms as an essential since they have all been encountered during the investigations so far done: Staphylococcus aureus, S. albus, Bacillus coli communis, B. proteus, B. fluorescens liquefaciens, B. fuorescens nonliquefaciens, B. Prodigiosus, and B. subtitis.

* G. Harold Drew, 'On the Precipitation of Calcium Carbonate in the Sea, etc.,
M.B.A., Vol. IX, No. 4, 1913 . 